Recepción: 18/ 09 / 2018

Aceptación: 16 / 11 / 2018

Publicación: 03 / 12 / 2018

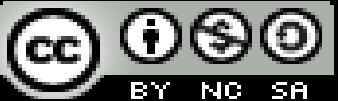

Ciencias económicas y empresariales

Artículo de investigación

\title{
Estrategia de Geomarketing para la cadena de Farmacias Cruz Azul en la ciudad Ambato-Ecuador
}

Geomarketing strategy for the Cruz Azul Pharmacy chain in the city AmbatoEcuador

\section{Estratégia de Geomarketing para a cadeia de Farmácia de Cruz Azul na cidade Ambato-Equador}

Carlos V. Mejía-Vayas ${ }^{\mathrm{I}}$ cv.mejia@uta.edu.ec

Lizbeth E. Bonilla-Segura ${ }^{\mathrm{II}}$ lbonilla815@gmail.com

Leonardo G. Ballesteros-López ${ }^{\text {III }}$ leonardogballesteros@uta.edu.ec

Cesar A. Guerrero-Velástegui IV ca.guerrero@uta.edu.ec

Correspondencia: cv.mejia@uta.edu.ec

\footnotetext{
I Magíster en Tecnología de la Información y Multimedia Educativa, Magíster Administración, Ingeniero en Empresas, Ingeniero de Sistemas y Computación, Docente de la Investigador Universidad Técnica de Ambato, Ambato, Ecuador.

${ }^{\text {II }}$ Docente de la Universidad Técnica de Ambato, Ambato, Ecuador.

III Magíster en Gestión Estratégica Empresarial MBA, Ingeniero Comercial, Licenciado en ciencias Administrativas Administrador en Mercadotecnia, Docente de la Universidad Técnica de Ambato, Ambato, Ecuador.

IV Magíster en Administración de Empresas Mención Planeación, Ingeniero de Empresas, Abogado de los Tribunales y Juzgados de la República del Ecuador, Docente de la Universidad Técnica de Ambato, Ambato, Ecuador.
} 


\title{
Resumen
}

El geomarketing en el ámbito empresarial analiza el comportamiento de los usuarios o clientes tomándose como referencia su localización, sus opiniones y valoración sobre un negocio o empresa. Así, el objetivo de esta investigación es proponer una estrategia de geomarketing para la cadena de farmacias Cruz Azul de la ciudad de Ambato-Tungurahua. El problema radica en que la cadena de farmacias Cruz Azul no realiza mediciones ni análisis del comportamiento de los clientes a través de herramientas de estadística espacial. Los resultados fundamentales es el desarrollo de una estrategia de geomarketing, a partir de una metodología que conlleve a establecer las fases y actividades, que se apoye en herramientas de estadística espacial y que contribuya a la reputación online de los establecimientos.

Palabras clave: geolocalización; geomarketing; estrategia de geomarketing; cadena de farmacias.

\begin{abstract}
Geomarketing in the business world analyzes the behavior of users or customers, taking as a reference their location, opinions and valuation of a business or company. Thus, the objective of this research is to propose a geomarketing strategy for the Cruz Azul pharmacy chain in the city of Ambato-Tungurahua. The problem lies in the fact that the Cruz Azul pharmacy chain does not perform measurements or analyze the behavior of customers through spatial statistics tools. The main results are the development of a geomarketing strategy, based on a methodology that leads to establishing the phases and activities, based on spatial statistics tools and that contributes to the online reputation of the establishments.
\end{abstract}

Keywords: geolocation; geomarketing; geomarketing strategy; pharmacy chain.

\section{Resumo}

O geomarketing no mundo dos negócios analisa o comportamento de usuários ou clientes, tomando como referência sua localização, opiniões e valorização de um negócio ou empresa. Assim, o objetivo desta pesquisa é propor uma estratégia de geomarketing para a rede de farmácias Cruz Azul, na cidade de Ambato-Tungurahua. O problema está no fato de a cadeia de farmácia Cruz Azul não realizar medições ou analisar o comportamento dos clientes por meio de ferramentas de estatísticas espaciais. Os principais resultados são o desenvolvimento de uma 
estratégia de geomarketing, baseada em uma metodologia que leva ao estabelecimento de fases e atividades, com base em ferramentas de estatísticas espaciais e que contribuem para a reputação online dos estabelecimentos.

Palavras chave: geolocalização; geomarketing; estratégia de geomarketing; cadeia de farmácias.

\section{Introducción}

Con el avance tecnológico constante, las empresas se ven obligadas a innovar y buscar otras estrategias de marketing. De esta forma, sin importar la ubicación de las personas, siempre estarán conectados con otras personas o empresas (García-Fernández, Fernández-Gavira, GálvezRuiz, Sánchez-Oliver, \& Grimaldi-Puyana, 2017); (Enciso-Quispe, Moreno, Yaguana, ZelayaPolicarpo, \& Quezada-Sarmiento, 2018). Por lo tanto, las estrategias de marketing deben asociarse y utilizarse conjuntamente con la tecnología y las diferentes herramientas que apoyan esta labor, con el fin de adaptarse a las nuevas exigencias de los consumidores y del mercado.

La geolocalización permite obtener información a través de una localización geográfica que genera grandes oportunidades en el desarrollo de productos y servicios innovadores que permitan crear valor a los clientes (Aguiar-Castillo, Beerli-Palacio, \& Pérez-Jiménez, 2016); (Beltrán López, 2016); (García-Fernández et al., 2017). Así, la geolocalización apoya a las empresas para que no solo puedan encontrarles físicamente, sino también de forma virtual.

Para (Enciso-Quispe et al., 2018), aplican la geolocalización en varias etapas: planificación, análisis y diseño, implementación y pruebas en un modelo de geolocalización para los Centros de Salud especializados en Pediatría y Medicina Familiar, a través de red móvil. También, (GarcíaFernández et al., 2017) utilizan la geolocalización como herramienta para conocer el comportamiento de los clientes de los centros de fitness. Por lo tanto, se observa la versatilidad de la geolocalización, pues no solo se puede aplicar en ámbitos de medicina y para centros de fitness, sino también, en ámbitos empresariales, para conocer el comportamiento de los clientes, sus necesidades, deseos, e incluso opiniones de la empresa o de los productos y servicios que ofrece al mercado.

A partir de ésta, el geomarketing es un sistema integrado por datos que analiza la situación de una empresa o negocio y sus clientes desde un punto de vista geográfico, espacial y de localización 
virtual que permite la toma de decisiones (Latour \& Le Floc'h, 2001; (Moreno, 2001); (Chasco, 2003); (Baviera-Puig, Buitrago-Vera, \& Rodríguez-Barrio, 2013); (Beltrán López, 2016). Además, ofrece una forma de analizar cuidadosa y metódicamente la ubicación de los consumidores objetivo para lograr una mayor rentabilidad, en donde las personas tienden a congregarse con otras similares en términos de ciertos factores que pueden determinar el consumo, como: estado social, composición del hogar y etnicidad (Baviera-Puig, Buitrago-Vera, \& Escriba-Pérez, 2016) Entonces, el geomarketing se puede utilizar para analizar datos y tomar decisiones, con el objetivo de satisfacer las necesidades y los deseos del consumidor mientras se obtiene un beneficio mutuo.

En ese sentido, (Smith \& Sánchez, 2003) agregan que para determinar una estrategia de marketing es fundamental el estudio de las variables sociodemográficas, económicas, psicográficas y de estilo de vida. El geomarketing tiene un gran potencial para desarrollar estrategias de marketing con las cuales tomar decisiones comerciales y empresariales basadas en el espacio virtual, ya que corresponden a varias técnicas de análisis social, económico y demográfico, desde el punto de vista geográfico y mediante la segmentación de los clientes potenciales que están ubicados en el mercado espacial (Chaco-Yrigoyen, 2006); (Cliquet, 2013); (Cross, Belich, \& Rudelius, 2015). Entonces, una estrategia de geomarketing debe involucrar varios aspectos que rodean a los clientes, como son: donde se ubican, sus ingresos, nivel socioeconómico, su personalidad, facilidades de compra y gustos.

Por lo tanto, si se considera la geolocalización como una herramienta de comunicación entre las personas, se observan tres elementos importantes (Beltrán, 2015). El emisor que es la persona o empresa que se ubica en algún punto y lanza un mensaje, el receptor que es la persona o empresa que recibe la información y el medio de que se dispone para la comunicación. Sin embargo, esta información no es unidireccional y estática, ya que el receptor tiene la capacidad de responder ante el mensaje del emisor (Rodríguez, 2010), convirtiéndose los usuarios en consumidores críticos con capacidad de crear opinión, invirtiéndose así la cadena de información y transformándose en emisores activos.

En ese sentido, al aplicar el geomarketing en el ámbito empresarial analiza el comportamiento de los usuarios o clientes tomándose como referencia su localización, sus opiniones y valoración 
sobre un negocio o empresa. Por ello, el objetivo de ésta investigación es proponer una estrategia de geomarketing para la cadena de farmacias Cruz Azul de la ciudad de Ambato-Ecuador.

Cabe agregar que, el $56.1 \%$ de la población ecuatoriana posee un celular inteligente (INEC, 2016), de los cuáles el $46.75 \%$ de los ecuatorianos tiene servicio de acceso a internet móvil, como señala la Agencia de Control de Telecomunicaciones (Arcotel, 2017) y el $18 \%$ de los consumidores utilizan la geolocalización para adquirir productos relacionados a viajes, turismo y artículos tecnológicos (Statista, 2018). Además, la empresa consultora de mercados (The Cocktail Analysis, 2013) añade que la geolocalización interesa por la utilidad en sus búsquedas en un $56 \%$. Razón por la cual, el geomarketing permite realizar mediciones y seguimiento a los clientes de la cadena de farmacias Cruz Azul, sobre sus opiniones, preferencias y comportamiento de compra.

Por otra parte, el problema que se identifica por observación directa es que la cadena de farmacias Cruz Azul no realiza mediciones ni análisis del comportamiento de los clientes a través de herramientas de estadística espacial, lo que conlleva a un desconocimiento de estas herramientas. Así, los resultados a obtener con este trabajo son el desarrollo de una estrategia de geomarketing, a partir de una metodología que conlleve a establecer las fases y actividades, que se apoye en herramientas de estadística espacial y que contribuya a la reputación online de los establecimientos.

\section{Metodología}

Dentro de la metodología que se utilizó, consta el análisis de documentos, libros y revistas que aporten al tema de investigación, conocer su evolución y llegar a las conclusiones más importantes. Similarmente, se utilizará herramientas de estadística espacial como Citymaps, Waze, Fourquare y Google Maps para realizar mediciones cuantitativas y cualitativas de las fotos publicadas por los usuarios, sus opiniones y valoraciones, que aporten datos sobre el comportamiento de los clientes. La población de estudio son 30 farmacias de la cadena Cruz Azul de la ciudad Ambato-Ecuador que aparecen en Google Maps, pues ya poseen puntos de geolocalización y a partir de éste se medirán en las otras herramientas. 


\section{Resultados}

Después de la bibliografía encontrada, se propone las siguientes fases para la estrategia de geomarketing: planificación, diagnóstico, presentación de resultados, análisis del segmento de mercado espacial, implementación de acciones, y seguimiento y evaluación.

\section{Planificación}

En esta fase se define el periodo de investigación y las herramientas a utilizar. En este estudio, el periodo de investigación es de seis meses desde febrero hasta marzo 2018. Y después de una evaluación de las herramientas de estadística espacial, se seleccionó: Citymaps, Waze, Fourquare y Google Maps.

\section{Diagnóstico}

En ésta fase, se realiza un diagnóstico preliminar las farmacias de la cadena Cruz Azul de la ciudad Ambato-Ecuador que poseen puntos de geolocalización. Para ello, se utilizaron las herramientas Google Maps (Ilustración 1), CityMaps2Go (Ilustración 2), Foursquare (Ilustración 3) y Waze (Ilustración 4).

Ilustración 1. Puntos de geolocalización de la cadena Cruz Azul Google Maps

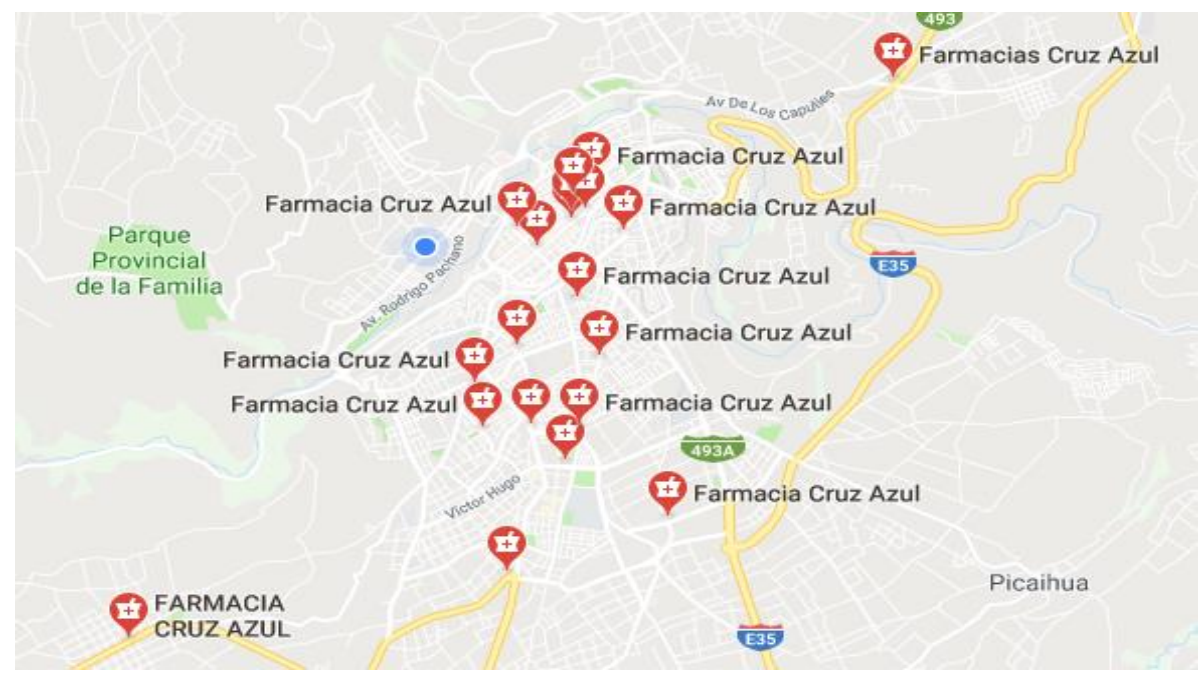




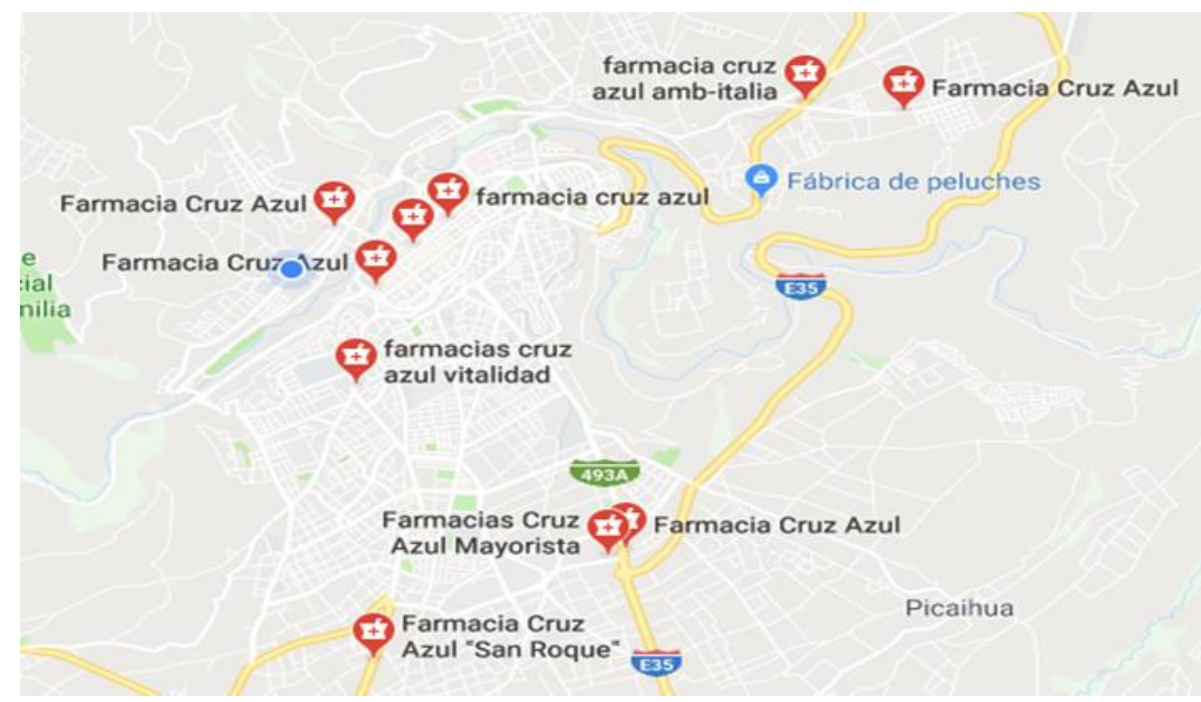

Fuente: (Google Maps, 2018)

Como se observa en la ilustración 1, se encontraron 30 puntos de geolocalización en el espacio virtual. Mismos que corresponden a las farmacias de la cadena Cruz Azul. Sin embargo, no todas las farmacias físicas se encuentran en este espacio virtual, puesto que no todos los locales poseen puntos de geolocalización, convirtiéndose en una desventaja.

Similarmente, en la ilustración 2, 3 y 4, se muestran algunos puntos de geolocalización, inferiores puntos que en la herramienta Google Maps. Entonces, se infiere que en estas herramientas CityMaps2Go, Foursquare y Waze no tienen presencia virtual; lo cual se debe aprovechar.

Ilustración 2. Puntos de geolocalización de la cadena Cruz Azul CityMaps2Go

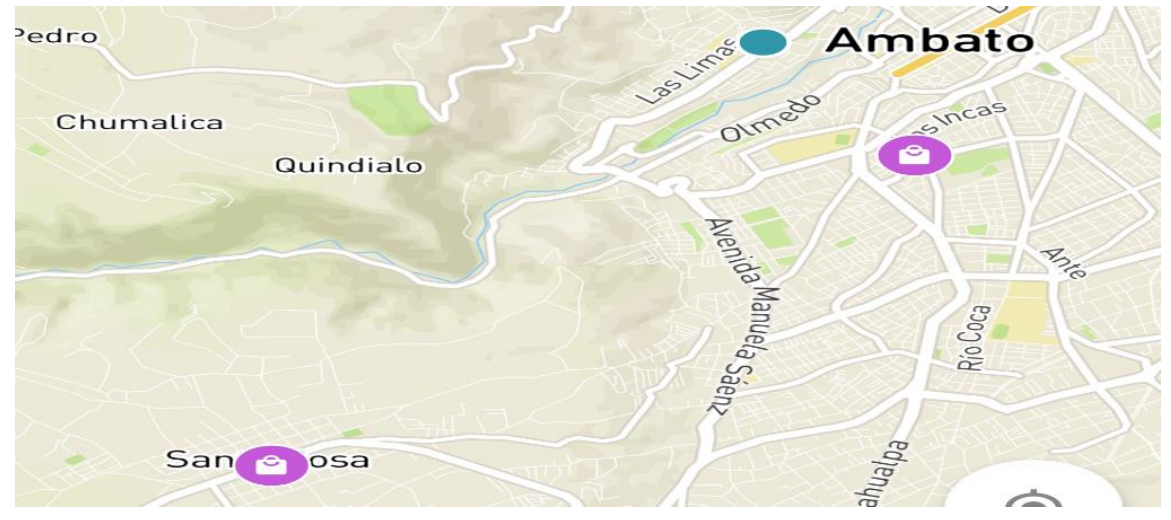

Fuente: (Ulmon, 2018) 
Ilustración 3. Puntos de geolocalización de la cadena Cruz Azul Foursquare

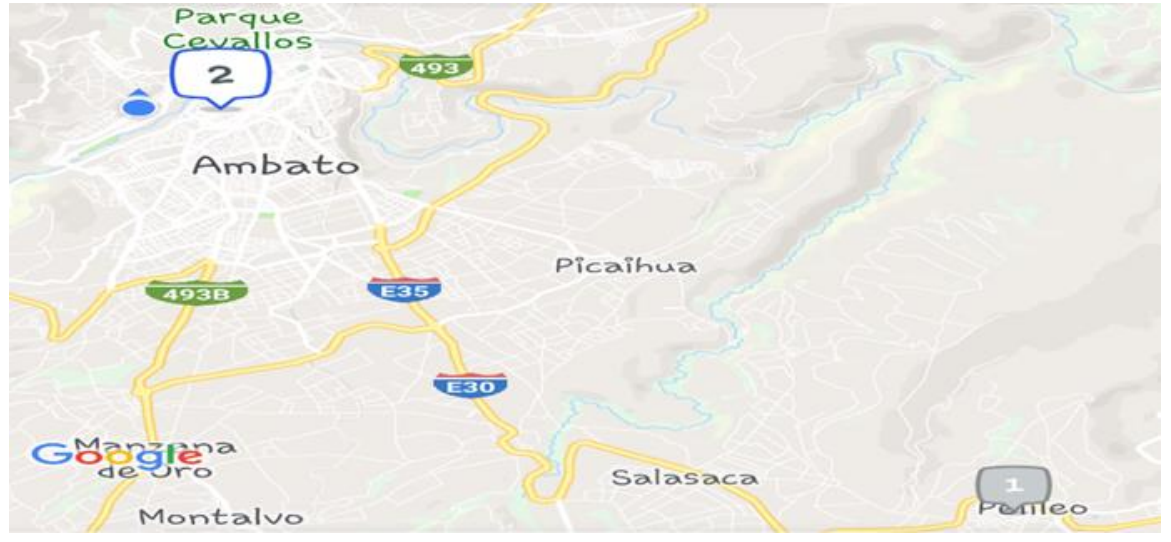

Fuente: (Foursquare, 2018)

Ilustración 4. Puntos de geolocalización de la cadena Cruz Azul Waze

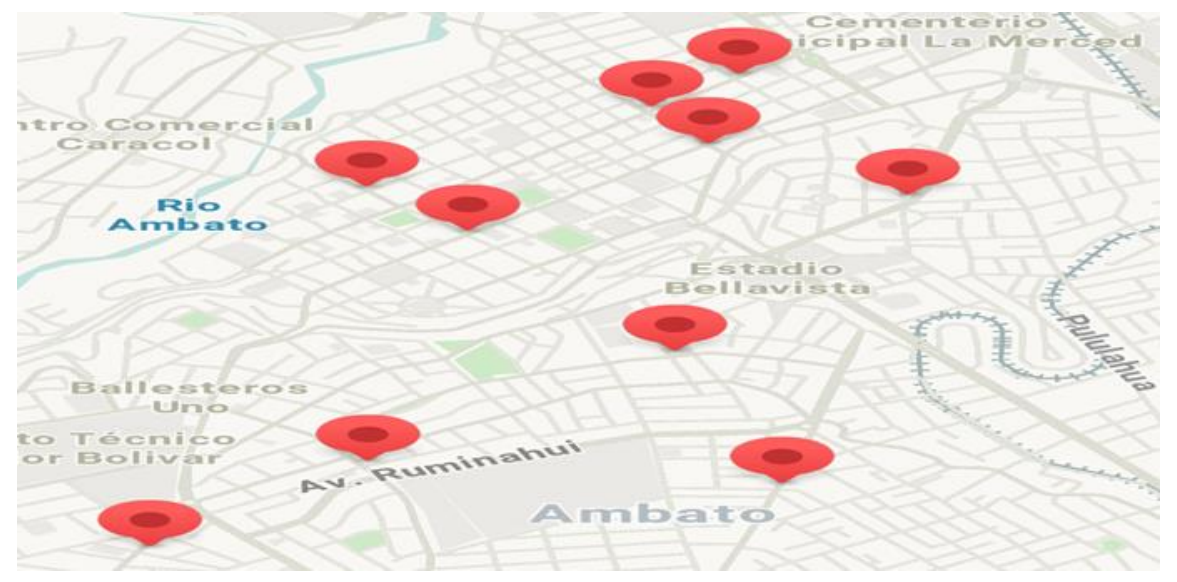

Fuente: (Waze, 2018)

Posteriormente, se recauda todas las farmacias con sus respectivos datos informativos (Ilustración $5)$. 
Ilustración 5. Farmacias de la cadena Cruz Azul Ambato-Ecuador

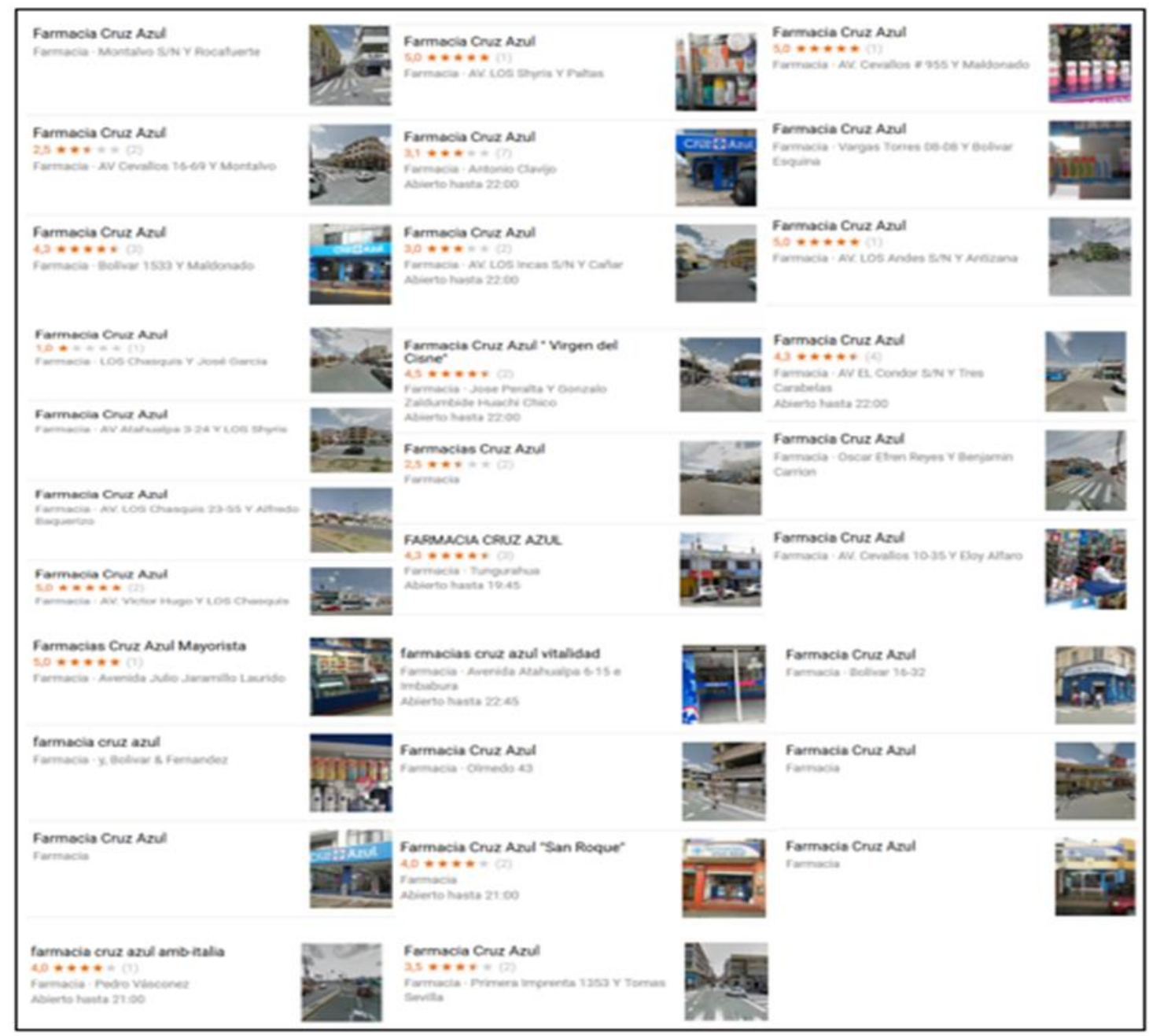

Fuente: (Google Maps, 2018)

\section{Presentación de resultados}

En ésta fase, se presentan los resultados encontrados después de una evaluación de las farmacias de la cadena Cruz Azul de la ciudad Ambato-Ecuador (Cuadro 1). 
Cuadro 1. Farmacias de la cadena Cruz Azul Ambato-Ecuador Google Maps

\begin{tabular}{|c|c|c|c|c|c|c|c|c|c|}
\hline \multirow[t]{2}{*}{ № } & \multirow[t]{2}{*}{ DIRECCIÓN } & \multirow{2}{*}{$\begin{array}{l}\text { PUNTUA- } \\
\text { CIÓN }\end{array}$} & \multicolumn{5}{|c|}{$\begin{array}{l}\text { PUNTACIÓN } \\
\text { ESTRELLAS }\end{array}$} & \multirow[t]{2}{*}{ RESEÑAS } & \multirow[t]{2}{*}{ FOTOS } \\
\hline & & & 1 & 2 & 3 & 4 & 5 & & \\
\hline 1 & $\begin{array}{l}\text { Av. Cevallos 16-69 y Montalvo, Ambato } \\
180101\end{array}$ & 2.5 & & 1 & & 1 & & 2 & 1 \\
\hline 2 & Bolívar $15-33$ y Maldonado, Ambato & 4.3 & & & 1 & 1 & 2 & 3 & 2 \\
\hline 3 & Antonio Clavijo, Ambato 180103 & 3.1 & 1 & 2 & 1 & 1 & 2 & 7 & 2 \\
\hline 4 & Av. Los Shyris y Paltas, Ambato 180202 & 5 & & & & & 1 & 1 & 2 \\
\hline 5 & Av. Los Incas y Cañar, Ambato 180202 & 3 & 1 & & & & 5 & 2 & 1 \\
\hline 6 & $\begin{array}{l}\text { Av. Cevallos 09-55 y Maldonado, Ambato } \\
180109\end{array}$ & 5 & & & & & 1 & 1 & 3 \\
\hline 7 & Av. Los Andes y Antizana, Ambato 180201 & 5 & & & & & 1 & 1 & 1 \\
\hline 8 & Los Chasquis y José Garcia, Ambato 180102 & 1 & 1 & & & & & 1 & 1 \\
\hline 9 & $\begin{array}{l}\text { Primera Imprenta } 13-53 \text { y Tomas Sevilla, } \\
\text { Ambato } 180109\end{array}$ & 3.5 & & 1 & & & 1 & 2 & 1 \\
\hline 10 & $\begin{array}{l}\text { AV. Víctor Hugo Y LOS Chasquis, Ambato } \\
180207\end{array}$ & 5 & & & & & 2 & 2 & 1 \\
\hline 11 & $\begin{array}{l}\text { José Peralta y Gonzalo Zaldumbide Huachi } \\
\text { Chico, Ambato } 180103\end{array}$ & 4.5 & & & & 1 & 1 & 2 & 1 \\
\hline 12 & $\begin{array}{l}\text { Av. EL Cóndor y Tres Carabelas, Ambato } \\
180205\end{array}$ & 4.3 & & & 1 & 1 & 2 & 4 & 1 \\
\hline 13 & Tungurahua, Santa Rosa & 4.3 & & & 1 & 1 & 2 & 3 & 3 \\
\hline 14 & Av. Indoamérica y Pedro Vásconez & 2.5 & 1 & & & 1 & & 2 & 1 \\
\hline 15 & Av. Julio Jaramillo Laurido, Ambato 180205 & 5 & & & & & 1 & 1 & 1 \\
\hline 16 & Calle Antonio Clavijo y Quisquis, Pelileo & 5 & & & & & 2 & 2 & 3 \\
\hline 17 & 22 DE Julio y Eloy Alfaro, Pelileo & 3 & & & 1 & & & 1 & 1 \\
\hline 18 & Garcia Moreno y 17 de Abril, Quero & 5 & & & & & 1 & 1 & 0 \\
\hline 19 & Av. Atahualpa y Gabriel García Mogrovejo & 4 & & & & 2 & & 2 & 2 \\
\hline 20 & Av. Indoamérica y Pedro Vásconez, Ambato & 4 & & & & 1 & & 1 & 1 \\
\hline 21 & Ambato, Baños de Agua Santa & 5 & & & & & 1 & 1 & 3 \\
\hline & SUMATORIA & 84 & 4 & 4 & 5 & 10 & 25 & 42 & 32 \\
\hline
\end{tabular}

Fuente: (Google Maps, 2018)

Por lo tanto, se muestra que de las 30 farmacias encontradas a través de la herramienta Google Maps, 21 de ellas poseen una puntuación de 84 puntos sobre 105. Lo que indica que la mayor parte de farmacias posee presencia en espacios virtuales. Sin embargo, ésta puntación es equivalente al número de reseñas o personas que han comentado y valorado sobre la atención y servicio de las farmacias. Por eso 42 personas han realizado algún tipo de comentario, sea positivo o negativo y asignado una valoración. De las cuales, 25 personas han asignado 5 
estrellas y 10 personas 4 estrellas, valores que reflejan la excelente atención, el stock de medicamentos para emergencias, la variedad y economía.

Cuadro 2. Farmacias de la cadena Cruz Azul Ambato-Ecuador CirtyMaps2Go y Foursquare

\begin{tabular}{|c|c|c|c|c|c|c|}
\hline \multicolumn{7}{|c|}{ CityMaps $2 G o$} \\
\hline $\mathbf{N}^{\mathbf{o}}$ & DIRECCIÓN & \multicolumn{3}{|c|}{$\mathbf{K M}$} & NOTAS & FOTOS \\
\hline 1 & Santa Rosa, Ambato & \multicolumn{3}{|c|}{5.2} & 0 & 0 \\
\hline 2 & Av. Los Shyris y Paltas & \multicolumn{3}{|c|}{1.5} & 0 & 0 \\
\hline \multicolumn{7}{|c|}{ Foursquare } \\
\hline \multirow{2}{*}{$\mathbf{N}^{\mathbf{o}}$} & \multirow{2}{*}{ DIRECCIÓN } & \multicolumn{3}{|c|}{ Valoración } & \multirow{2}{*}{ TIPS } & \multirow{2}{*}{ FOTOS } \\
\hline & & $\begin{array}{c}\text { Me } \\
\text { gusta }\end{array}$ & $\begin{array}{l}\text { Está } \\
\text { Bien } \\
\end{array}$ & No me gusta & & \\
\hline 1 & Padre Chacón, Pelileo & 1 & & & 1 & 0 \\
\hline 2 & $\begin{array}{l}\text { Mariano Castillo y Av } \\
\text { Cevallos }\end{array}$ & & 1 & & 0 & 0 \\
\hline & SUMATORIA & 1 & 1 & 0 & 1 & 0 \\
\hline
\end{tabular}

Fuente: (Ulmon, 2018); (Foursquare, 2018)

De acuerdo al cuadro 2, se muestra que las farmacias no utilizan este tipo de herramientas para su ubicación en espacio virtual, puesto que poseen valores mínimos. Razón por la cual, el desarrollo de una estrategia de geomarketing es fundamental.

Cuadro 3. Farmacias de la cadena Cruz Azul Ambato-Ecuador Waze

\begin{tabular}{|c|l|c|c|c|}
\hline \multirow{2}{*}{$\mathbf{N}^{\mathbf{0}}$} & \multicolumn{1}{|c|}{ DIRECCIÓN } & KM & \multicolumn{2}{c|}{ ESTACIONAMIENTO } \\
\cline { 5 - 5 } & & 0.9 & $\mathbf{S O C A L}$ & CERCA \\
\hline 1 & Av. Rodrigo Pachano y La Delicia & 1.1 & $\mathrm{No}$ & $\mathrm{Si}$ \\
\hline 2 & Juan Montalvo y Rocafuerte & 1.5 & $\mathrm{No}$ & $\mathrm{Si}$ \\
\hline 3 & Av. Cevallos y Juan Montalvo & 1.5 & $\mathrm{No}$ & $\mathrm{Si}$ \\
\hline 4 & Maldonado y Bolívar & 1.6 & $\mathrm{No}$ & $\mathrm{No}$ \\
\hline 5 & Bolívar y Vargas Torres & 1.7 & $\mathrm{Si}$ & $\mathrm{No}$ \\
\hline 6 & Av. El Rey y Los Andes & 1.5 & $\mathrm{No}$ & $\mathrm{Si}$ \\
\hline 7 & Cañar y Av Los Incas & 1.7 & $\mathrm{Si}$ & $\mathrm{Si}$ \\
\hline 8 & Av. Los Chasquis y José García & 2.1 & $\mathrm{Si}$ & $\mathrm{Si}$ \\
\hline 9 & Av. Los Shyris y Paltas & 1.9 & $\mathrm{Si}$ & $\mathrm{Si}$ \\
\hline 10 & Av. Antonio Clavijo y Natalia Vaca & 3.1 & $\mathrm{Si}$ & $\mathrm{Si}$ \\
\hline 11 & Av. Víctor Hugo, Ambato & & \\
\hline
\end{tabular}

Fuente: (Waze, 2018) 
En el cuadro 3, se observa que las farmacias de la cadena Cruz Azul poseen mayor presencia virtual en esta herramienta. Se detalla también los kilómetros desde donde se ubican los usuarios y si poseen estacionamientos dentro del local o cerca de ellos, valioso si los consumidores poseen vehículo o se trasladan en taxi o bus.

\section{Análisis del segmento de mercado espacial}

En esta fase, se debe especificar las variables sociodemográficas, económicas, psicográficas y de estilo de vida, de los clientes de las farmacias de la cadena Cruz Azul, con el fin de segmentar a los clientes potenciales que están ubicados en el mercado espacial.

Edad: personas entre 18-35 años.

Ingresos: de $\$ 386$ en adelante

Nivel socioeconómico: medio y medio alto

Beneficios buscados: rapidez en la compra, economía, garantía y calidad en los medicamentos.

Modalidad de compra: compra física en los locales.

Estilos de vida: personas que gustan de informarse por internet, y gustan de la tecnología y específicamente, redes sociales y aplicaciones móviles.

Personalidad: personas con una vida ágil, extrovertidas y que emitan sus criterios.

\section{Implementación de acciones}

En esta fase se detallan las estrategias de geomarketing que a consideración de la autora se deben poner en práctica para obtener mejores resultados en la reputación de la cadena de farmacias Cruz Azul.

Estimar la demanda potencial de cada farmacia de la cadena.

Dirigir en cada farmacia un producto estrella, que se promocione en el espacio virtual.

Ejecutar planes de marketing basado en geomarketing.

Ubicar más punto de geolocalización en las herramientas. 
Utilizar los storytelling basado en mapas para conocer los criterios e historias de la marca y del servicio.

Subir más fotos en las herramientas, para que los usuarios interactúen con estas.

Incrementar la tasa de visita en las aplicaciones móviles, con campañas de publicidad para que los clientes conozcan a través de que aplicaciones pueden encontrarlos y valorar su servicio.

\section{Seguimiento y evaluación.}

En esta etapa, las farmacias deberían realizan un seguimiento y evaluación esta estrategia cada cuatro meses, puesto que poseen muchos locales que no poseen puntos de geolocalización en las herramientas. Además, para evaluar esta estrategia se detallan a continuación las fórmulas (cuadro 4).

Cuadro 4. Seguimiento y evaluación d las cadenas de Farmacias

\begin{tabular}{|c|c|c|}
\hline Nombre & Fórmula & Descripción \\
\hline Tasa de conversión/visitas & $\begin{array}{l}\text { Cantidad de ventas } \\
\text { Número de visitas } \\
\text { a las aplicaciones }\end{array}$ & $\begin{array}{l}\text { Conocer si los clientes } \\
\text { utilizan las aplicaciones para } \\
\text { evaluarlos después de la } \\
\text { compra. }\end{array}$ \\
\hline Nivel de servicio post-venta & $\frac{\text { Comentarios respondidos }}{\text { Comentarios realizados }}$ & $\begin{array}{l}\text { Analizar el nivel de servicio } \\
\text { y atención al cliente, para } \\
\text { conocer cómo influyen en } \\
\text { expectativas del cliente y } \\
\text { captar problemas } \\
\text { operacionales. }\end{array}$ \\
\hline Valoración del usuario & $\frac{\text { Valoración de los usuarios }}{\text { Total valoración usuarios }}$ & $\begin{array}{l}\text { Analizar el servicio y } \\
\text { atención al cliente en cada } \\
\text { uno de los locales. }\end{array}$ \\
\hline
\end{tabular}

Fuente: elaboración propia

\section{Conclusiones}

Esta investigación permitió una fundamentación teórica y metodológica sobre en la geolocalización y el geomarketing, las cuales permitieron la determinación de elementos clave en las fases de la estrategia. 
Además, las estrategias de marketing deben asociarse y utilizarse conjuntamente con la tecnología y las diferentes herramientas que apoyan esta labor, con el fin de adaptarse a las nuevas exigencias de los consumidores y del mercado. En ese sentido, el geomarketing se puede utilizar para analizar datos y tomar decisiones, con el objetivo de satisfacer las necesidades y los deseos del consumidor mientras se obtiene un beneficio mutuo. Involucrándose aspectos que rodean a los clientes, como son: donde se ubican, sus ingresos, nivel socioeconómico, su personalidad, facilidades de compra y gustos. Y a partir de ésta información se estructuró cada una de sus fases, dando como resultado seis fases para la estrategia de geomarketing.

\section{Referencias bibliográficas}

Aguiar-Castillo, L., Beerli-Palacio, A., \& Pérez-Jiménez, R. (2016). La geolocalización: Oportunidades de marketing para el hotel del futuro (Vol. 9, pp. 375-388). Presentado en XI Congreso internacional de Turismo y Tecnologías de la Información y la Comunicación, España: TURITEC 2016.

Arcotel. (2017). Boletín Estadístico Unificado Junio 2017. Recuperado de http://www.arcotel.gob.ec/wp-content/uploads/2015/01/BOLETIN-ESTAD\%C3\%8DSTICOUNIFICADO-JUNIO-2017v3.pdf

Baviera-Puig, A., Buitrago-Vera, J., \& Escriba-Pérez, C. (2016). GEOMARKETING MODELS IN SUPERMARKET LOCATION STRATEGIES. Journal of Business Economics and Management, 17(6), 1205-1221. https://doi.org/10.3846/16111699.2015.1113198

Baviera-Puig, A., Buitrago-Vera, J., \& Rodríguez-Barrio, J. E. (2013). Un modelo de geomarketing para la localización de supermercados: diseño y aplicación práctica. DOCFRADIS, $1-30$.

Beltrán López, G. (2016). Geolocalización online: La importancia del dónde. Barcelona, España: Editorial UOC.

Chaco-Yrigoyen, C. (2006). Análisis estadístico de datos geográficos en geomarketing: el programa GeoDa [Statistical analysis of geographic data in marketing: the program GeoDa]. Distribución y Consumo, 16(86), 34-47. 
Chasco, C. (2003). El geomarketing y la Distribución Comercial. Investigación y marketing, 79(6-13).

Cliquet, G. (2013). Geomarketing:Methods and Strategies in Spatial Marketing. London, UK: John Wiley \& Sons.

Cross, J. C., Belich, T. J., \& Rudelius, W. (2015). How marketing managers use market segmentation: an exploratory study. (pp. 531-536). Presentado en In Proceedings of the 1990 Academy of Marketing Science (AMS) Annual Conference, Cham, Switzerland: Springer International Publishing.

Enciso-Quispe, L., Moreno, S., Yaguana, J., Zelaya-Policarpo, E., \& Quezada-Sarmiento, P. A. (2018). A geolocation model of health centers specialized in pediatrics and family medicine using the concept of mobile network. En 2018 13th Iberian Conference on Information Systems and Technologies (CISTI) (pp. 1-6). Caceres: IEEE. https://doi.org/10.23919/CISTI.2018.8399313

Foursquare. (2018). Foursquare. Recuperado de https://es.foursquare.com/

García-Fernández, J., Fernández-Gavira, J., Gálvez-Ruiz, P., Sánchez-Oliver, A. J., \& GrimaldiPuyana, M. (2017). La Geolocalización como Herramienta para Conocer a los Consumidores: El Caso de los Centros de Fitness. PODIUM Sport, Leisure and Tourism Review, 6(2), 263-276. https://doi.org/10.5585/podium.v6i2.228

Google Maps. (2018). Google Maps. Recuperado de https://www.google.com/maps

INEC. (2016). Tecnologías de la Información y Comunicaciones (TIC’s) 2016. Recuperado de http://www.ecuadorencifras.gob.ec/documentos/webinec/Estadisticas_Sociales/TIC/2016/170125.Presentacion_Tics_2016.pdf

Latour, P., \& Le Floc'h, J. (2001). Géomarketing: Principes, méthodes et applications. París, Francia: Éditions d'Organisation.

Moreno, A. (2001). La geografía de los servicios aplicada al marketing como tema de estudio. Madrid, España: Universidad Autónoma de Madrid y Asociación de Geógrafos Españoles. 
Smith, L. D., \& Sánchez, S. M. (2003). Assessment of business potential at retail sites: empirical findings from a US supermarket chain. International Review of Retail, Distribution and Consumer Research, 13(1), 37-58.

Statista. (2018). Ofertas de geolocalización y productos adquiridos 2015. Recuperado 24 de marzo de 2018, de https://es.statista.com/estadisticas/501251/productos-y-servicios-adquiridostras-el-uso-de-servicios-de-geolocalizacion-en-espana/

The Cocktail Analysis. (2013). V Estudio sobre Mobile Marketing de IAB Spain y The Cocktail Analysis. Recuperado de http://www.infoadex.es/home/wpcontent/uploads/2017/12/RESUMEN-2013.pdf

Ulmon. (2018). City Maps 2Go. Recuperado de http://www.ulmon.com/

Waze. (2018). Waze. Recuperado de https://www.waze.com/es-419 